\title{
Bilingüismo y desplazamiento del español. Notas sobre el caso de Tijuana, B. Cfa.
}

\author{
Lourdes Gavaldón de Barreto \\ Centro de Estudios Lingüísticos y Literarios \\ El Colegio de México
}

\section{Resumen}

Nos proponemos efectuar un estudio poniendo en práctica los recursos que ha aportado la sociolingüística en los últimos años para conocer y describir con mayor objetividad la relación de lenguas en contacto que se desarrolla en Tijuana. Tomando lo anterior como base, esta investigación proporcionará una visión de conjunto sobre los principales mecanismos que entran en juego, los que determinan la tendencia a seguir, los que indican los cambios cualitativos en los procesos, y sobre todo, la relación que guardan entre ellos. De esta manera, será posible conocer la interacción de los factores económicos, políticos, sociales y culturales que influyen en la realización lingüística, así como determinar los niveles de la lengua: fonológico, morfológico, sintáctico así como el léxico. Con ello la comunicación verbal se convierte en un indicador que permite identificar los factores sociales que predominan y condicionan los cambios.

Palabras clave: sociedad bilingüe, lingüística, ciudades fronterizas, desplazamiento del español, emigrados.

\begin{abstract}
Our objective is to start a research using the resources of socio-linguistics in order to discover and describe in a more objective form the relationship of contact languages developed in Tijuana. Based on the before mentioned, this research will offer a global vision of the main mechanisms that take part, those that determine the trends to follow and, at mean time to show the qualitative changes of the processes; but more specifically, the relationship existing between them. In this way, we will be able to learn how the economical, political, social, and cultural factors interact affecting the linguistic performance, as well as to determine the diverse levels of language: phonological, morphological, syntax, as well as lexical. In this way, oral communication becomes an indicator that allows us to identify the social factors that prevail and condition changes.
\end{abstract}

Keywords: bilingual society, linguistics, border cities, displacement of Spanish language, emigrant. 


\title{
BILINGÜISMO Y DESPLAZAMIENTO DEL ESPAÑOL NOTAS SOBRE EL CASO DE TIJUANA, B. CFA.
}

\author{
Por: \\ Lourdes Gavaldón de Barreto \\ Investigadora del Centro de Estudios Lingüisticos y \\ Literarios de El Colegio de México
}

La interacción de las culturas mexicana y americana se lleva a cabo en forma incuestionable a lo largo y a lo ancho de ambos territorios nacionales. Sin embargo, es la frontera entre los dos países donde se efectúan los procesos socio-culturales más dinámicos. Su importancia se manifiesta en muchas direcciones y con diferente grado de alcance. Estudios sobre tan compleja relación son necesarios, para conocer, por lo menos, sus características más importantes y orientar su desarrollo.

A la fecha no hay ningún estudio sobre las condiciones en las que se realiza la interacción lingüística en la zona fronteriza entre México y los Estados Unidos, a pesar de que se ha resaltado en diversas ocasiones su trascendencia a nivel local y a nivel nacional. ${ }^{1}$

En realidad, es una situación que se desconoce casi por completo. No ha gozado de atención alguna, no obstante que para calificar esta circunstancia se menciona un estado de alarma ya que se piensa que el inglés influye en la frontera mexicana con tal fuerza que pone en peligro la integridad del español en esta zona. Además, seguramente por la proximidad geográfica con los Estados Unidos, tiende a identificarse con el estado que guarda el español en territorio americano, donde las condiciones de desarrollo varfan radicalmente. $^{2}$

Nos hemos propuesto efectuar un estudio poniendo en práctica los recursos que ha aportado la sociolinguiística en los últimos años para conocer y describir con mayor objetividad la relación de lenguas en contacto que se desarrolla en Tijuana.

1

Por ejemplo, en la mayor parte de las ponencias lefdas durante el Simposio Nacional sobre Estudios Fronterizos organizado por El Colegio de México y la Facultad de Filosofía y Letras de la Universidad de Nuevo León en Monterrey, N.L. del 24 al 27 de Enero de 1979.

2

Las múltiples manifestaciones del español en los Estados Unidos han atraído la atención de diversos especialistas, en consecuencia, existe un buen número de estudios que tratan sobre este tema. Cfr. Teschner, Richard Garland, D. Bills and Jerry A. Craddock, Spanish and English of United States Hispanos. A critical, annotated, linguistic bibliography. Center por Applied Lingüistic, Arlington. 1975, $352 \mathrm{pp}$. 
Una investigación construída sobre dichas bases proporcionará una visión de conjunto: sobre los principales mecanismos que entran en juego, los que determinan la tendencia a seguir, los que indican los cambios cualitativos en los procesos, y, sobre todo, la relación que guardan entre ellos. De esta manera, será posible conocer la interacción de los factores económicos, políticos, sociales, culturales, etc., que influyen en la realización lingüística. También desde el punto de vista de la lengua, en este caso el español, para determinar el nivel de lengua: el fonológico, el morfológico, el sintáctico o el léxico que es más susceptible al cambio. Con ello, la comunicación verbal se convierte en un indicador constante y explícito a tal grado que permite identificar aquellos factores sociales que predominan sobre otros y condicionan los cambios. ${ }^{3}$

Como punto de partida, para ubicar nuestro estudio sobre bilingüismo y desplazamiento del español en Tijuana, haremos una breve mención, en primer lugar, a los rasgos politicos y sociales que describen en general a la población.

La localización geográfica de la ciudad de Tijuana ha influido en forma determinante en su desarrollo. Se encuentra situada en el ángulo formado hacia el oeste por el Oceano Pacífico y hacia el norte por la línea fronteriza que la separa del condado de San Diego, Calif. Es el punto más alejado de la República Mexicana, y, a excepción de Mexicali y Ensenada, se encuentra aislada de los demás centros nacionales de población. Esto se debe principalmente a la gran distancia que media entre la zona norte del estado de Baja California y el resto del territorio nacional. Baste mencionar que entre Tijuana y la ciudad de México hay que recorrer actualmente unos 3000 kms. por carretera.

Desde un punto de vista histórico, los primeros asentamientos urbanos en la región tuvieron lugar con posterioridad y a consecuencia de los Tratados de Guadalupe Hidalgo. Se reconoce el año de 1889 como fecha de fundación de Tijuana. Sin embargo, a pesar de su corta vida, ha experimentado un acelerado crecimiento. Esto se atribuye principalmente a que Tijuana es la localidad mexicana más cercana a la rica y numerosa población del sur de California. Desde su inicio, ha gozado de la preferencia de 3

Si bien hay otros factores que igualmente son indicadores de los rasgos propios de cada cultura, tales como, el sistema familiar, los valores morales, tradiciones ceremoniales, para mencionar algunos, los beneficios que ofrece la realización lingürstica para la investigación y el conocimiento de la sociedad son limitados. Baste pensar en la riqueza de sus elementos y en la frecuencia de uso por parte de los miembros de cada cultura. 
los norteamericanos a un grado tal, que se ha convertido en el centro turístico más visitado por ellos. Por la puerta de San Ysidro cruzaron en el año de 1978, 34 millones de personas de Tijuana a San Diego. ${ }^{4}$

La proximidad con los Estados Unidos, aunado a los salarios más altos de la República, ha atraf́lo a una innumerable cantidad de personas del interior del país desde hace varias décadas a esta ciudad. En 1930, la localidad tenía tan solo 11,271 pobladores, y, cincuenta años fíás tarde, en 1980, fueron censadas 800000 personas. $^{5}$ Es la quinta ciudad de la nación de acuerdo al número de habitantes, y, en términos urbanos, es la ciudad de mayor atractivo e índice de crecimiento del pars. ${ }^{6}$

Los estados de Jalisco y Sonora, principalmente, son los que han enviado los contingentes más numerosos, pero prácticamente todos los estados del país están representados aquí. La constante llegada de nuevos pobladores le da a la ciudad una característica de mosaico regional.

La cercanfa con los Estados Unidos ha propiciado una situación peculiar. Pues si bien el turismo norteamericano representa la fuente principal para la generación de divisas de la ciudad, la dependencia económica de la población para satisfacer sus necesidades en todos los órdenes es exageradamente elevada.

Esta dependencia económica no se refiere tan solo al abastecimiento de los bienes materiales de consumo, sino que ha trascendido a los más diversos órdenes: a las relaciones laborales que se establecen con los trabajadores fronterizos, los indocumentados, los obreros de las maquiladoras, las relaciones comerciales, el turismo fronterizo, los espectáculos y diversiones, las relaciones familiares entre chicanos y mexicanos, la transmisión directa de los medios de comunicación masiva, para mencionar algunos.

Tijuana tiene enfrente, como un gran escaparate, a la tercera ciudad en importancia del estado más rico del país más rico del mundo. En consecuencia, los términos de comparación son negativos para Tijuana en muchos sentidos de tal suerte que se pierde de vista la etapa histórica por la cual está pa-

\section{4}

San Ysidro Port of Entry is the busiest land border crossing point in the world, Economic Analysis of Mexican Citizen Activity in San Diego County. San Diego Chamber of Commerce, San Diego, 1979 , p. 3 .

5

Según informes extraoficiales, a través de la prensa, de la Dirección General del Censo. 6

Cf. Unikel, Luis, El Desarrollo Urbano de México. El Colegio de México, México 1976, Cuadro I-A3. 
sando cada uno de estos dos países. Por ésto, los marcos de referencia se restringen a una situación fronteriza en desventaja para esta ciudad. Generalmente se ignora que, a nivel nacional, la localidad posee indices satisfactorios en cuanto a la calidad de vida. ${ }^{7}$

Debido a esta proximidad con los Extados Unidos, observamos que e] inglés se practica constantemente en Tijuana en las relaciones comerciales con los turistas y en el trato de negocios con los americanos. De esta manera, encontramos una manifestación explícita de las relaciones de las dos cul. turas a través de la comunicación verbal. Sabemos que la realización linguirs. tica de los habitantes de la ciudad varfa desde un monolingüismo en español. un bilingüismo español-inglés, a un desplazamiento del español por el inglés. Pero no podemos precisar qué clase de bilingüismo se practica, cómo se inte. gra a la vida diaria de la población, si efectivamente hay un desplazamientc del español a causa del inglés y cuál es su alcance. Estas interrogantes son las que inicialmente nos propusimos examinar dentro del contexto general de la población.

Para definir el concepto de bilingüismo, lenguas en contacto y desplazamiento de una lengua, hemos considerado la experiencia en otras regiones de bi- o multilingüismo en el mundo. Parece ser que no se aplica ninguna regla constante en cuanto a esta situación. Por ejemplo, puede ser que predomine la lengua del grupo cultural más débil frente a las de uno más fuerte, o a la inversa. Un conocido autor, Joshua Fishman menciona en diferentes obras ${ }^{8}$ ejemplos que ilustran varias posibilidades: coexistencia, rechazo, pérdida de una de las dos lenguas, en una generación, en dos generaciones, particularización de cada una de las lenguas según el tema que se esté tratando, según los interlocutores, etc.

Otro autor, Glyn Lewis, en su estudio sobre el multilingüismo en la Unión Soviética ${ }^{9}$, distingue varios tipos de bilingüismo característicos de? desplazamiento de alguna de las lenguas, y establece diferencias tomando en cuenta factores extralingüísticos. Mencionaremos algunos de estos tipos de bilingüismo:

7

Como atención médica, Indice de escolaridad y casa habitación por habitante, etc. Cfr. Price, John, Tijuana: Urbanization in a Border Culture, University of Notre Dame Press. Notre Dame, Indiana, 1973, p. 25. 8

Fishman, Joshua, Bilingual Education, Newbury House Publisher, Inc. Massachusetts, 1977, p. 5 Cf. del mismo autor, Language and Sociocultural Change. Stanford University Press, Stanford, Calif. 1972.

9

Lewis, Glyn, Multilingualism in the Soviet Union, Mouton, The Hague, 1972, pp. 278, 282. 
Bilingüismo estable: Aquel que está caracterizado por la coexistencia de grupos linguísticos geográficamente contiguos. En este caso los hablantes bilingües pasan indiferentemente de una lengua a otra, ante cualquier tema, dominio o actitud.

Bilingüismo dinámico: Se produce entre las personas que están en constante cambio en la escala social o en el medio geográfico, es decir, en función de la movilidad social o del cambio de lugar. Se convierte en una necesidad introducir la lengua de la otra clase social o de la nueva zona geográfica para facilitar el cambio hacia la asimilación. Este tipo de bilingüismo sí crea una especialización que excluye sistemáticamente una de las dos lenguas, según el dominio el tema $u$ otras circunstancias. La necesidad de asimilarse a los patrones de la nueva sociedad obliga a los inmigrantes a sustituir la lengua original por la del país receptor.

Bilingüismo oral frente a bilingüismo letrado: Cuando las personas hablan, leen o escriben ambas lenguas, es posible mantener un bilingüismo estable. En caso contrario, se manifiesta una inseguridad en el uso de aquella que se conoce en menor grado, y el hablante tenderá a desplazarla por la que le cuesta menor esfuerzo. Por ello, se creará también una especialización por dominios, temas, etc., en el uso de una de las dos.

De la clasificación de Lewis resulta evidente que hay condiciones de relación social que implican un desplazamiento y otras una coexistencia. En suma, el bilingüismo oral se convierte, al cabo de corto tiempo, en un monolingüismo. Solo el bilingüismo estable admite la coexistencia de los dos sistemas por tiempo indefinido, mientras se mantengan las mismas condiciones socioculturales. Por lo tanto, no todo bilinguiismo está condenado a desplazar a la lengua materna, más bién está sujeto a eventualidades de orden social, económico y político principalmente.

Sin embargo, el bilinguiismo es la condición necesaria para llegar a un desplazamiento y pérdida de la lengua original. Un punto intermedio es lo que se ha llamado interferencia. Uriel Weinreich lo define en estos términos: Le terme d'interference implique un nouvel arrangement des structures par suite de l'introduction d'élements étrangers dans les domaines plus fortement structurés d'une langue, tels que l'ensemble du systéme des phonémes, une grande partie de la morphologie et de la syntax, et certaines sphéres du vocabulaire ${ }^{10}$. La interferencia se manifiesta ampliamente en el es10

Weinreich, Uriel, Langues en contact, Publications du Cercle Linguistique de New York, No. 1 New York, 1953, p. 1. 
pañol chicano de los Estados Unidos. Rosaura Sánchez lo caracteriza como la experiencia más sobresaliente de la expresión chicana ${ }^{11}$. No se trata de préstamos léxicos que se adaptan al sistema de la lengua receptora, sino que son cambios dentro de la cadena hablada con modificaciones fonológicas, morfológicas, sintácticas o léxicas o todas juntas. Por ejemplo: Iba a controlar el City Hall y el School Board, Puede dar better results si lo saben usar, You had a baile, Nomás anda fooling around ${ }^{12}$.

En cierta forma el fenómeno de la interferencia hace que dos sistemas linguísticos diferentes se fundan en uno solo de manera irregular con respecto a la clase de elementos o a las partes de la oración que se van a conmutar. Esta situacion no se prolonga indefinidamente sino que aunado a la problemática social que motivó la interferencia resulta en la eliminación de una de las dos lenguas.

\section{Situación linguiística de Tijuana}

Después de revisar estas experiencias teóricas, comenzamos a elaborar un método de acercamiento a la situación de Tijuana. Como un primer intento para conocer la actitud de los hablantes del lugar respecto al uso, preferencia y valoración del español y del inglés, elaboramos un cuestionario de aplicacion directa que tomara en cuenta estos temas. Es evidente que un análisis basado en la actitud es relativo, pues lo que se piensa sobre el tema y su realización efectiva no coinciden necesariamente. Por otra parte, la presencia del encuestador condiciona en forma incontrolable y en sentido diferente a los informantes. Sin embargo, aún tomando en cuenta estas limitaciones, puede ser útil para conocer una parte del proceso.

Con este cuestionario de aplicación directa, y, a manera de sondeo, llevamos a cabo 25 entrevistas con informantes de la ciudad. El objetivo era conocer su punto de vista y determinar cuáles factores habría que tomar en consideración desde su punto de vista para completar nuestra investigación sobre bilingüismo y desplazamiento del español en Tijuana. Esta muestra no puede considerarse representativa de la localidad en términos estrictos, pues el número y las caracteristicas de los informantes no fueron calculados en ninguna proporción respecto a la ciudad. Sólo fueron controladas las siguientes variables:

11

Sánchez Rosaura, Chicano Code-Switching en Swallow VI, San Diego State University, San Diego, S.F. p. 179.

12

Ibid., p.p. $177-193$ 
a) sexo: mitad de hombres y mitad de mujeres,

b) generación: el grupo comprendido entre los 18 y 55 años de edad.

c) permanencia: con un mínimo de 5 años de residir en la localidad,

ch) actividades: una muestra que incluyera la mayor diversidad de ocupaciones,

d) clase sociali en términos generales que estuvieran representadas tres clases sociales: baja, media y alta;

e) lugar de habitación: que residieran en diversas secciones de la ciudad.

Se aplicó un cuestionario completo a cada informante. El cuestionario tiene un promedio de 90 preguntas. Estas preguntas se refieren a datos personales del informante, su estructura familiar, lugar de origen, tiempo de residencia en esta ciudad, colonia donde habita, escolaridad, actividad, ingreso, contacto con la frontera mexicana, conocimiento y práctica del inglés del lado americano y en México, clase de contacto con anglohablantes, contacto pasivo con el inglés, grado de conocimiento del inglés, casos de interferencia, juicios de valor sobre la situación de lenguas en contacto en Tijuana etc. Se hicieron preguntas adicionales en los casos en que surgía espontáneamente una información interesante. Se buscaron respuestas de carácter amplio que aportaran datos. En cada caso, se pedía la colaboración de la persona elegida y en ese momento se aplicaba el cuestionario. De este modo, no se adoptaron actitudes muy pensadas de antemano. Cada una de las encuestas se grabó en cinta magnetofónica, y ninguna excedió de tres cuartos de hora a la velocidad de $33 / 4$.

A continuación nos referiremos a algunos resultados obtenidos de la muestra de 19 informantes: Sobre el lugar de origen, sólamente tres habłan nacido en la localidad, cuatro en Jalisco, dos en el Distrito Federal, dos en Sinaloa, dos en Baja California, dos en los Estados Unidos, uno en Durango, uno en Coahuila y uno en Sonora. Siete personas habran llegado antes de los 15 años y 9 después de los 15 años. En cuanto a la zona de residencia, vivían en 15 sectores diferentes de la ciudad. Todos habían cruzado la frontera en alguna ocasión. La escolaridad se manifestó de la siguiente manera: seis habran cursado algún año de primaria, ocho habían terminado la primaria, y habłan estudiado carrera comercial, alguna especialización técnica o secundiaria: los seis restantes eran profesionistas o estudiantes de la universidad. Con respecto al inglés, tres dijeron que hablaban un poco y ocho afirmaron que sí lo hablaban.

Coincidieron todos los informantes en cuanto a la realización del inglés en los siguientes puntos: 
a) en Tijuana se hablaba inglés o se oía hablar inglés en las zonas turísticas,

b) ninguno hablaba en inglés en el seno familiar o en la relación social entre hispanohablantes,

c) los que si hablaban inglés lo usaban cuando había un interlocutor anglohablante que no hablara español, fuera en la relación familiar, social, comercial o turística,

ch) consideran que aprender inglés representa un conocimiento recomendable que permite mejores oportunidades de trabajo,

d) que en Tijuana se habla un español muy contaminado, que no saben expresarse correctamente. Hay ideales imprecisos de pureza del idioma español.

Al relacionar algunos datos proporcionados por los informantes podemos anticipar algunas conclusiones. Observamos que de las tres personas nacidas en Tijuana, dos no hablan inglés; de las ocho que no hablan inglés, seis son mujeres; de las ocho que si hablan inglés, siete llegaron antes de los quince años; cinco informantes de los ocho que no hablan inglés, llegaron después de los 15 años. La muestra es muy interesante, parece apuntar ciertas tendencias, como la importancia de la edad al llegar a Tijuana, así como el sexo. Sin embargo, es prematuro dar por seguros tales resultados.

Sin duda alguna, el sondeo aportó, por lo menos, una experiencia de contacto directo con los habitantes. No obstante, aumentar la muestra de manera que fuera representativa de la población nos indicaría las tendencias con mayor seguridad. En suma, el cuestionario fue útil, también, para identificar la relacion de ciertas variables para continuar investigando en esa dirección.

Lo que sí podemos considerar como un resultado evidente es la ausencia de casos de interferencia, aunque esté sujeto a comprobación en una muestra más amplia. Llegamos a esta conclusión desde dos puntos de vista. En primer lugar no encontramos ejemplos de interferencia en el contexto mismo de las respuestas de los informantes. En segundo lugar, cuando preguntamos por qué está contaminado el español de Tijuana, la respuesta general fue que se usan términos del inglés como pari (party "fiesta"), bilera (bill of notes: "cuaderno de notas") tail (tile: "mosaico"), tuneo (tune up "afinación de automóviles"), señal (signal "semáforo"). Estos ejemplos son casos de préstamos léxicos, no se trata de interferencia propiamente dichas, tal como las definimos. Esta manifestación refleja una actitud consciente de distinción entre los elementos de la expresión verbal. Aún una gran parte de los préstamos léxicos que se toman del inglés se identifican como extraños. 
Después de escuchar las cintas, nos percatamos que hay varios grupos diferentes de habitantes en su relación con los Estados Unidos. Aún no sabemos cuál es la importancia cuantitativa de cada uno de ellos, sí de su influencia en el contexto general de la población, sin embargo, el comportamiento linguístico de cada uno de estos grupos determina en gran medida las características del proceso de lenguas en contacto de Tijuana. Por ahora nos concretaremos a destacarlos:

a) los que viven en Tijuana y no participan directamente de la cercanía con los Estados Unidos,

b) los que viven en Tijuana y se benefician del comercio, diversiones, etc., en territorio norteamericano,

c) los que viven en Tijuana pero que tienen un familiar económicamente activo en los Estados Unidos. Este familiar puede cruzar la frontera a diario, los fines de semana, ciertas épocas del año, cada año;

ch) los que han vivido largo tiempo en los Estados Unidos pero actualmente residen en Tijuana.

Hay que hacer mencion de otro grupo que ha llamado poderosamente la atención por la importancia humana, política y económica que representa para los dos paises; el de los trabajadores indocumentados. Parece ser que la opinión pública en general identifica la mayor parte de los habitantes de Tijuana con los trabajadores indocumentados. En realidad, en proporciones que aún no podemos precisar, la gran mayoría de los trabajadores indocumentados constituyen una poblacion flotante en la ciudad; permanecen corto lapso de tiempo mientras usan la ciudad de Tijuana como trampolín al estado de California. Investigadores como Jorge Bustamante ${ }^{13}$ y Wayne Cornelius ${ }^{14}$, señalan que estados como Zacatecas, Guanajuato, Chihuahua, Michoacán y Jalisco proporcionan el mayor número de trabajadores indocumentados, y nos los sitúan como oriundos de Baja California. Cierto es que la llegada de un trabajador a Tijuana ofrece varias alternativas: intentar cruzar la frontera, tener éxito e internarse en los Estados Unidos para quedarse allí definitivamente o bien regresarse al cabo de cierto tiempo a su lugar de origen; fracasar en los innumerables intentos, permanecer en Tijuana "mientras" lo logra, y convertirse en un residente; o bien, traer a su familia, dejarla en Tijuana, para cruzar la frontera periódicamente.

13

Bustamante, Jorge, Indocumentados, Mitos y Realidades, El Colegio de México.México, 1978, p. 34. 14

Cornelius, Wayne, Mexican and Caribbean Migration to the United States: The Staie of Current Knowledge and Recommendations for future Research. University of California in San Diego, San Diego, p. 88. 
La gran mayoría de los indocumentados cruza la frontera o se regresa a su lugar de origen sin dejar huella en la ciudad. El pequeño grupo restante el que permanece en Tijuana, es el que va a pasar por el proceso de adaptación a esta ciudad al igual que otros grupos de inmigrantes.

Sin duda alguna, cada uno de estos grupos de habitantes está expuesto en diferente medida al bilingüismo. Sin embargo, solamente los que se quedan a residir en forma permanente en los Estados Unidos están presionados activamente a asimilarse a los patrones norteamericanos.

Los habitantes de Tijuana tienen una opción de márgenes mas flexibles. Pero ¿quiénes de ellos se inclinarán conscientemente por un bilingüismo estable? y ¿quiénes por un desplazamiento del español? ¿cuáles serán las condiciones extralingüisticas que motiven activamente este cambio? ¿sobre todo? ¿cuál es la trascendencia de esta actitud linguística en el contexto general de la población en cuanto a valores de la cultura mexicana?.

Los resultados obtenidos durante este primer intento de acercamiento están aún incompletos para poder dar por seguras las conclusiones formuladas. No obstante, pueden considerarse como una base para el estudio de las lenguas en contacto de la ciudad de Tijuana: su condicionamiento, su perspectiva de desarrollo y sus consecuencias. 